\title{
A Possible Mechanism for Renoprotective Effect of Sodium-Glucose Cotransporter 2 Inhibitor: Elevation of Erythropoietin Production
}

\author{
Hidekatsu Yanai ${ }^{a}$, , Hisayuki Katsuyayama ${ }^{a}$
}

\section{To the Editor}

We read a recent review on the renoprotective effect of sodiumglucose cotransporter 2 inhibitor (SGLT2i) [1], with a great interest. Sano et al mentioned that SGLT2i reduces the overload of the proximal tubules and improves tubulointerstitial hypoxia, inducing the recovery of erythropoietin production by fibroblasts [1]. They concluded that increased hematocrit during SGLT2 $i$ therapy indicates the recovery of tubulointerstitial function in diabetic kidney [1]. Elevation of hematocrit was also observed in the Empagliflozin Cardiovascular Outcome Event Trial in Type 2 Diabetes Mellitus Patients (EMPAREG OURCOME) [2], which showed the reduction of risk of major adverse cardiovascular events by empagliflozin in patients with type 2 diabetes. Recently, EMPA-REG OUTCOME demonstrated that the use of empagliflozin was associated with slower progression of kidney disease as compared with placebo [3]. However, the underlying mechanisms for the renoprotective effect of SGLT2i remain largely unknown.

Graphed results of our previous study showing the effects of six kinds of SGLT2i (dapagliflozin, ipragliflozin, tofogliflozin, luseogliflozin, canagliflozin and empagliflozin) on metabolic parameters were shown in Figure 1 [4]. Hematocrit promptly increased at 1 month after the use of SGLT2i, and gradually increased until 6 months after the use of SGLT2i (Fig. 1a). Estimated glomerular filtration rate (eGFR) showed a short-term decrease from baseline to month 1 , however, gradually increased from month 1 to month 3 , and remained stable from month 3 to month 6 (Fig. 1b). The change in eGFR after the use of SGLT2i in our study was closely similar to the change in eGFR observed in EMPA-REG OUTCOME [3]. Further, our data supported Sano's hypothesis that increased hematocrit during SGLT2i therapy indicates the recovery of

Manuscript accepted for publication December 08, 2016

a Department of Internal Medicine, National Center for Global Health and Medicine Kohnodai Hospital, Chiba, Japan

${ }^{\mathrm{b}}$ Corresponding Author: Hidekatsu Yanai, Department of Internal Medicine, National Center for Global Health and Medicine Kohnodai Hospital, 1-7-1 Kohnodai, Ichikawa, Chiba 272-0034, Japan.

Email: dyanai@hospk.ncgm.go.jp

doi: https://doi.org/10.14740/jocmr2857w tubulointerstitial function in diabetic kidney [1].

The authors of EMPA-REG OUTCOME listed a decrease in hyperfiltration and glomerular hypertension, and effects on arterial stiffness, vascular resistance, serum uric acid and the systemic and renal neurohormonal systems as the mechanisms for renoprotective effects of empagliflozin [3]. We think that elevated erythropoietin which increases hematocrit may be also a possible mechanism for the renoprotective effect of SGLT2i. We previously reported a case of type 2 diabetes with severe peripheral artery disease and mild microangiopathy, whose serum erythropoietin level was significantly elevated $(58.6 \mathrm{mU} / \mathrm{mL}$ (normal range: $8-36 \mathrm{mU} / \mathrm{mL}$ )) [5]. The ankle-brachial pressure index decreased significantly (right: 0.82 , left: 0.84 ) and a computed tomography angiogram of the leg revealed bilateral severe atherosclerosis and stenosis of the distal branch of popliteal arteries. However, she did not show overt proteinuria and urinary albumin excretion was very small (30.2 mg/day).

Chronic treatment with recombinant human erythropoietin exerted renoprotective effects beyond hematopoiesis in streptozotocin-induced diabetic rat [6]. Erythropoietin protected mouse podocytes from damage by advanced glycation end-products [7]. Furthermore, erythropoietin ameliorated podocyte injury in advanced diabetic nephropathy in the $\mathrm{db} /$ $\mathrm{db}$ mouse [8]. In humans, serum erythropoietin transiently increased from baseline in the dapagliflozin group up to week 4, followed by a gradual decline until week 12 [9].

In conclusion, increased production of erythropoietin induced by SGLT2i may be one of the mechanisms which can explain the renoprotective effect of SGLT2i.

\section{Conflicts of Interest}

The authors declare that they have no conflicts of interest concerning this article.

\section{References}

1. Sano M, Takei M, Shiraishi Y, Suzuki Y. Increased Hematocrit During Sodium-Glucose Cotransporter 2 Inhibitor Therapy Indicates Recovery of Tubulointerstitial Function in Diabetic Kidneys. J Clin Med Res. 2016;8(12):844- 

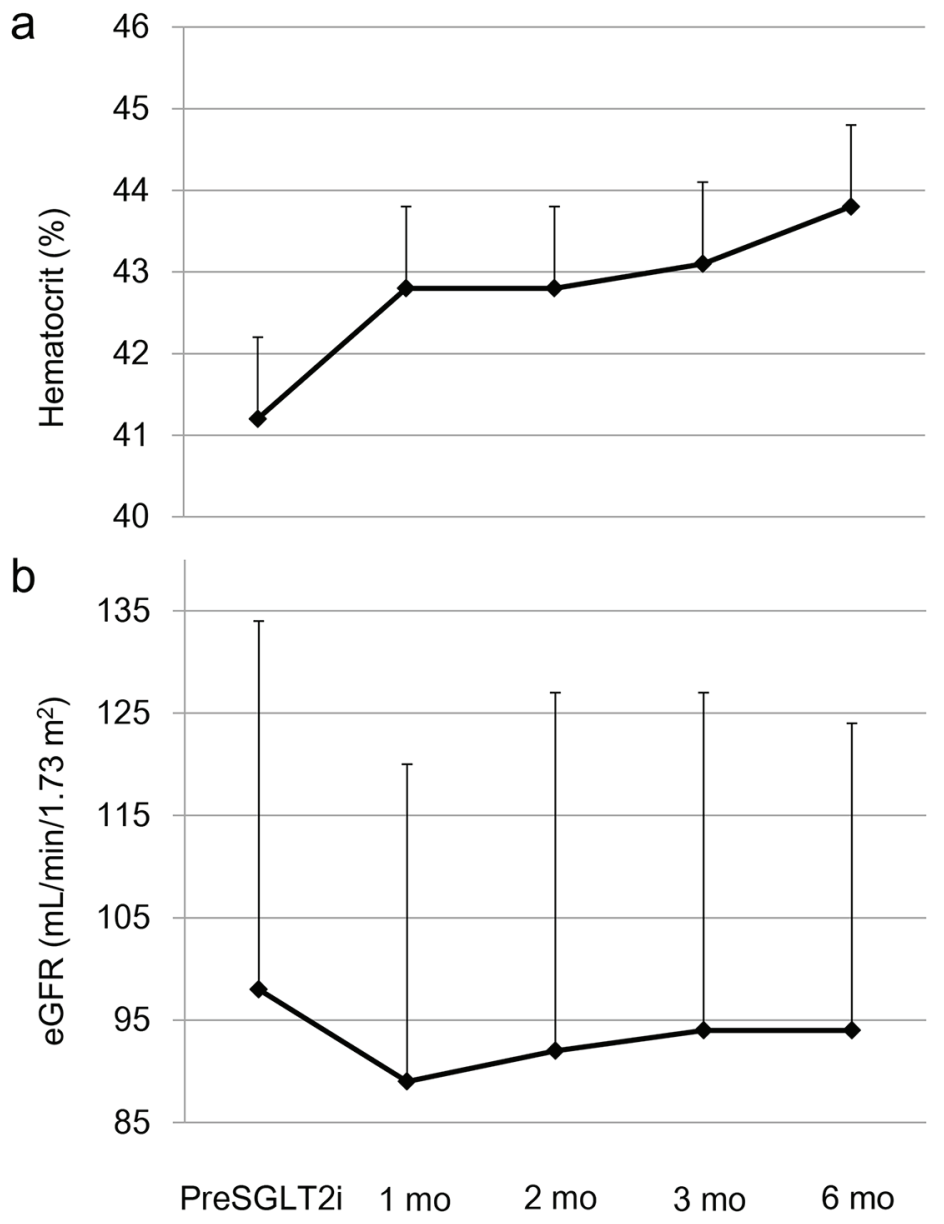

Figure 1. The changes in (a) hematocrit and (b) estimated glomerular filtration rate (eGFR) at 1,2, 3 and 6 months after the start of sodium-glucose cotransporter 2 inhibitor (SGLT2i). This figure shows the graphed results of our previous study [4]. PreSGLT2i indicates before the start of SGLT2i. Boxes and bars indicate mean and SD.

847.

2. Zinman B, Wanner C, Lachin JM, Fitchett D, Bluhmki E, Hantel S, Mattheus M, et al. Empagliflozin, Cardiovascular Outcomes, and Mortality in Type 2 Diabetes. N Engl J Med. 2015;373(22):2117-2128.

3. Wanner C, Inzucchi SE, Lachin JM, Fitchett D, von Eynatten M, Mattheus M, Johansen OE, et al. Empagliflozin and Progression of Kidney Disease in Type 2 Diabetes. N Engl J Med. 2016;375(4):323-334.

4. Katsuyama H, Hamasaki H, Adachi H, Moriyama S, Kawaguchi A, Sako A, Mishima S, et al. Effects of Sodium-Glucose Cotransporter 2 Inhibitors on Metabolic Parameters in Patients With Type 2 Diabetes: A Chart-Based Analysis. J Clin Med Res. 2016;8(3):237-243.

5. Hamasaki H, Moriyama S, Yanai H. A crosstalk between macroangiopathy and microangiopathy in type 2 diabetes. Int J Cardiol. 2013;168(1):550-551.
6. Toba H, Sawai N, Morishita M, Murata S, Yoshida M, Nakashima K, Morita Y, et al. Chronic treatment with recombinant human erythropoietin exerts renoprotective effects beyond hematopoiesis in streptozotocin-induced diabetic rat. Eur J Pharmacol. 2009;612(1-3):106-114.

7. Ruester C, Franke S, Bondeva T, Wolf G. Erythropoietin protects podocytes from damage by advanced glycation end-products. Nephron Exp Nephrol. 2011;117(1):e2130.

8. Loeffler I, Ruster C, Franke S, Liebisch M, Wolf G. Erythropoietin ameliorates podocyte injury in advanced diabetic nephropathy in the $\mathrm{db} / \mathrm{db}$ mouse. Am J Physiol Renal Physiol. 2013;305(6):F911-918.

9. Lambers Heerspink HJ, de Zeeuw D, Wie L, Leslie B, List J. Dapagliflozin a glucose-regulating drug with diuretic properties in subjects with type 2 diabetes. Diabetes Obes Metab. 2013;15(9):853-862. 